\title{
Diagnostic Capacity for Tuberculosis in Various Laboratories within Meru County, Kenya
}

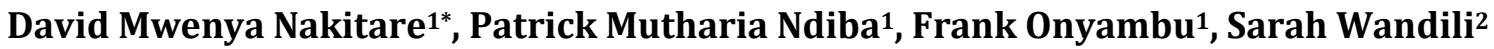 \\ ${ }^{1}$ School of Health Sciences, Meru University of Science and Technology, Meru, Kenya \\ ${ }^{2}$ Department of Biological Sciences, Meru University of Science and Technology, Meru, Kenya \\ Email: ^davidnakitare@gmail.com
}

How to cite this paper: Nakitare, D.M., Ndiba, P.M., Onyambu, F. and Wandili, S. (2019) Diagnostic Capacity for Tuberculosis in Various Laboratories within Meru County, Kenya. Journal of Tuberculosis Research, 7, 238-245.

https://doi.org/10.4236/jtr.2019.74023

Received: October 4, 2019

Accepted: December 16, 2019

Published: December 19, 2019

Copyright $\odot 2019$ by author(s) and Scientific Research Publishing Inc. This work is licensed under the Creative Commons Attribution International License (CC BY 4.0).

http://creativecommons.org/licenses/by/4.0/

c) (i) Open Access

\begin{abstract}
Background: The Division of Leprosy, Tuberculosis and Lung Disease reported a notification rate of 217/100,000 in Meru County. This study aimed at determining diagnostic capacity for tuberculosis in various medical laboratories within Meru County. Objective: To determine the diagnostic capacity for tuberculosis in various laboratories within Meru County. Materials and Methods: This was a cross-sectional survey which utilized quantitative techniques to establish the potential and capacity of TB diagnostic laboratories in selected medical institutions. Observational checklist was used to determine essential requirements for sample processing. Data entry was done through use of Statistical Package for Social Sciences (SPSS). Data analysis was done using Chi-square. Results: Results indicated that there was significant difference in laboratory diagnostic capacity at $\left[\chi^{2}(1 \mathrm{df}, \mathrm{N}=26)=18.49, \mathrm{p}=0.000\right.$ $<0.05]$ for Florescence microcopy and $\left[\chi^{2}(1 \mathrm{df}, \mathrm{N}=26)=18.49, \mathrm{p}=0.000<\right.$ 0.05] for Gene Xpert Assay. There were significant differences in the diagnostic capacity of TB between public and private/commercial laboratories in relation to availability of the resources. Conclusion: Public operated laboratories had enhanced Tuberculosis (TB) diagnostic capacity as compared to the private laboratories.
\end{abstract}

\section{Keywords}

Diagnostic Capacity, Tuberculosis

\section{Introduction}

Tuberculosis (TB) is an airborne disease caused by Mycobacterium tuberculosis. It is an infection which typically affects the pulmonary system and can also affect other parts of the body [1]. It is spread by cough droplets through the release 
tubercle bacilli from people who are infected with pulmonary TB. A majority of people in the world are exposed to TB but a relatively small proportion of people infected with M. tuberculosis develop TB disease [1]. A number of factors are known to influence susceptibility to TB disease including sex, nutrition, being in low income groups, HIV infection, living in congested environments such as prisons ${ }^{1}$, even though $\mathrm{TB}$ is a major opportunistic infection among people infected with HIV. Mycobacterium tuberculosis is a non-motile, non-spore-forming and obligate aerobic bacterium. Transmission of pulmonary tuberculosis disease by M. tuberculosis accounts for $98 \%$ of all tuberculosis cases [2] [3]. The replication of the tubercle bacilli takes place in alveolar macrophages once an individual is infected. The severity of TB disease is highly based on the emergence of chest pain and coughing of bloody sputum [2] [3]. Laboratories which are privately owned do carry out sputum smear microscopy test on suspected TB patients. In order to strengthen their testing capacity, the national standard operating procedures and training materials for tuberculosis should be used for day to day operations, thus providing data to the National Leprosy and Tuberculosis Program (NLTP) [4] [5].

The new reported cases of tuberculosis in 2013 were estimated at 9.0 million and 1.5 million deaths. The number of people who died of TB as an opportunistic infection in HIV/AIDS patients was estimated at 0.4 million. In the same year, 3.3 million cases and 510,000 TB deaths had occurred among women as well as an estimated 550,000 cases and 80,000 deaths among children [6]. The expansion of TB services through introduction of new tests including Drug susceptibility testing (DST), Polymerase Chain Reaction (PCR)-based test and Gene-Xpert MTB/RIF came in beside acid-fast bacillus smear microscopy in diagnosing TB in Human Immunodeficiency Virus-associated TB as well as drug-resistant TB to optimally control TB through World Health Organization's approach [7].

Kenya is one of the 22 countries with high TB burden in the world. In the year 2014, the Kenyan population was estimated at 45 million while estimates of TB burden 2014, MDR-TB burden 2014 were as follows; RR-/MDR-TB 2014, TB/HIV 2014 and Laboratories 2014 were tabulated [8] [9].

It was therefore important to determine the diagnostic capacity for tuberculosis in selected laboratories within Meru County.

\section{Materials and Methods}

\section{Study site}

The survey was carried out within Meru municipality ward in Meru County in eastern Kenya.

\section{Study population}

The study population involved laboratory technicians and technologists working in selected medical laboratories within Meru municipality as well as medical laboratories within Meru municipality ward. All laboratory technicians 
and technologists working in the medical laboratories in Meru Municipality were included in the study. The study purposively selected laboratories including Meru Teaching and Referral Hospital, Agakhan hospital, Karen Hospital, Gakoromone dispensary, Marrie-stopes clinic, Family Care Medical Centre, New southlands clinic, Kinoru dispensary, Woodlands hospital, Eastern consultants clinic, Milimani Maternity and Nursing Home and Meru town clinic. The laboratory managers were asked to state the number of laboratory personnel working in their respective laboratories and it was as follows; Meru Teaching and Referral Hospital $=18$, Gakoromone dispensary $=2$, Agakhan hospital $=1$, Karen Hospital $=2$, Marrie-stopes $=1$, Family Care Medical Centre $=1$, New southlands clinic $=1$, Kinoru dispensary $=4$, Woodlands hospital $=1$, Eastern consultants clinic $=2$, Milimani Maternity and Nursing Home $=1$, and Meru town clinic $=1$.

\section{Sampling procedure}

Stratified random sampling technique was utilized to obtain the study sample. The first process involved dividing the population into smaller groups based on the health institutions that they came from thus forming a stratum. In comparison to the population, a random sample from each stratum was taken with a number proportional to the size of the stratum. These subsets of the strata were pooled to form a random sample. In this study, 26 Laboratory technicians and technologists, which is a $74.3 \%$ of the total Laboratory technicians and technologists, were selected to participate in the study. Stratified random sampling technique was used to select 26 health care workers from the 12 selected medical laboratories within Meru Municipality ward. Stratified random sampling ensured that all the individual respondents had an equal and independent chance of being selected as a member of the sample (Table 1).

\section{Criteria for inclusion and exclusion}

The study population involved laboratory technicians and technologists working in selected medical laboratories within Meru municipality as well as medical laboratories within Meru County in Meru municipality ward. All laboratory technicians and technologists working in the medical laboratories within Meru Municipality in Meru County were included in the study. In addition, laboratory personnel who gave informed consent to participate in the study were included. Medical laboratories within Meru municipality were also included in the study.

\section{Data extraction}

Semi-structured questionnaires were administered to laboratory technicians and technologists in selected laboratories. Inspection checklist was also used and collected data focusing on availability of equipment, reagents and fume chamber for sputum processing within the laboratories setting. The process was coordinated to ensure that the data collection process was properly done. Ethical clearance was obtained from the institutional review board.

\section{Statistical analysis}

Data was entered, coded and cleaned in the SPSS. Frequency and percentages were calculated. Findings were presented in form of frequency distribution tables and bar graphs and analyzed using Chi square. 
Table 1. Proportionate distribution of the sample.

\begin{tabular}{cccc}
\hline Laboratory & $\begin{array}{c}\text { Total Laboratory } \\
\text { Technicians/ } \\
\text { Technologists }\end{array}$ & $\begin{array}{c}\text { \% Population } \\
\text { of all Technicians/ } \\
\text { Technologists }\end{array}$ & $\begin{array}{c}\text { Sampled } \\
\text { Technicians/ } \\
\text { Technologists }\end{array}$ \\
\hline $\begin{array}{c}\text { Meru Teaching } \\
\text { and referral } \\
\text { Milimani Maternity \& } \\
\text { Nursing home }\end{array}$ & 18 & 51.4 & 14 \\
Gakoromone Dispensary & 2 & 2.9 & 1 \\
Meru Town clinic & 1 & 5.7 & 1 \\
New Southlands & 1 & 2.9 & 1 \\
Family care & 1 & 2.9 & 1 \\
Medical Centre & 1 & 2.9 & 1 \\
Marrie-stopes & 1 & 2.9 & 1 \\
Agakhan hospital & 2 & 2.9 & 1 \\
Karen Hospital & 2 & 5.7 & 1 \\
Eastern Consultants & 1 & 5.7 & 2 \\
Woodlands Hospital & 4 & 2.9 & 26 \\
Kinoru dispensary & 35 & 11.4 & 1 \\
Total & & $100 \%$ & 1 \\
\hline
\end{tabular}

\section{Results}

\section{Laboratory category}

The study sought information on the different categories of laboratories which existed in Meru Municipality. The information generated from this item is presented in Table 2.

Findings in Table 2 illustrate that majority of respondents had indicated that their laboratories were public laboratories (69.2\%) followed by private/commercial laboratories (30.8\%). This implies that majority of laboratories were from the public health facilities which are government owned thus enabling diagnostic capacity for TB.

Differences in diagnostic capacity amongst the laboratories in relation to availability of resources

Table 3 presents findings on the differences in the Tuberculosis diagnostic capacity amongst the laboratories in relation to availability of resources.

There existed no significant differences among the laboratories in terms of availability of microscopes, access to electricity and availability of sink with running water. This was so because all the laboratories in the study had a microscope, access to electricity and had a sink with running water. There were also no statistical significant differences among the laboratories in terms of availability of availability of Thermo cycler machines since these machines were missing in all the studied laboratories in Meru Municipality. However there were significant differences in the diagnostic capacity of TB between public and private/commercial laboratories in relation to availability of the following re- 
sources: Safety cabinet $\left[\chi^{2}(1 \mathrm{df}, \mathrm{N}=26)=18.49, \mathrm{p}=0.000<0.05\right]$, Centrifuge $\left[\chi^{2}(1 \mathrm{df}, \mathrm{N}=26)=10.64, \mathrm{p}=0.000<0.05\right]$, Gene Xpert resources $\left[\chi^{2}(1 \mathrm{df}, \mathrm{N}=\right.$ $26)=18.49, \mathrm{p}=0.000<0.05]$, Freezer/refrigerator $\left[\chi^{2}(1 \mathrm{df}, \mathrm{N}=26)=14.28, \mathrm{p}=\right.$ $0.000<0.05]$, Incubator [ $\left.\chi^{2}(1 \mathrm{df}, \mathrm{N}=26)=18.49, \mathrm{p}=0.000<0.05\right]$ and Auramine $\mathrm{O}$ reagents for TB florescence microscopy $\left[\chi^{2}(1 \mathrm{df}, \mathrm{N}=26)=18.49, \mathrm{p}=\right.$ $0.000<0.05$ ] (see appendix V). The public or government operated facilities or laboratories had enhanced TB diagnostic capacity as compared to the private/commercial laboratories.

Referral of specimens to a TB reference laboratory for culture and sensitivity

The study sought information on whether they referred specimens for TB culture and sensitivity to TB reference laboratories. The information generated from this item is presented in Table 4.

The findings in Table 3 illustrate that majority of respondents had been referring specimens for TB culture and sensitivity to reference laboratories (73.1\%) while those who were not referring them $(26.9 \%)$. This implies that majority of the respondents were referring specimens for culture and sensitivity which is a good practice in diagnostic capacity for TB in Health facilities. There were significant differences in relation to referring TB specimens to a TB reference laboratory for culture and sensitivity between the public and private laboratories $\left[\chi^{2}(1 \mathrm{df}, \mathrm{N}=26)=21.55, \mathrm{p}=0.000<0.05\right]$. Public laboratories made more referrals to a TB reference laboratory making them to have enhanced TB diagnostic capacity as compared to private/commercial laboratories.

\section{Reference laboratories for TB culture and sensitivity specimen}

The study sought information on reference laboratories where they had been sending specimen for TB culture and sensitivity. The information generated from this item is presented in Table 5.

Table 2. Laboratory category.

\begin{tabular}{ccc}
\hline Laboratory category & Number of laboratories & Percentage (\%) \\
\hline Public laboratory & 18 & 69.2 \\
Private/commercial laboratory & 8 & 30.8 \\
Total & 26 & 100.0
\end{tabular}

Table 3. Differences in TB diagnostic capacity amongst laboratories in relation to availability of resources.

\begin{tabular}{ccccc}
\hline Availability of laboratory resources & $\chi^{2}$ Value & Df & N & Sig (p-value) \\
\hline Safety cabinet & 18.49 & 1 & 26 & 0.000 \\
Centrifuge & 10.64 & 1 & 26 & 0.001 \\
Gene Xpert resources & 18.49 & 1 & 26 & 0.000 \\
Freezer/refrigerator & 14.28 & 1 & 26 & 0.000 \\
Incubator & 18.49 & 1 & 26 & 0.000 \\
Auramine O reagents for & 18.49 & 1 & 26 & 0.000 \\
TB florescence microscopy & & & & \\
\hline
\end{tabular}


Table 4. Referral of specimens to a TB reference laboratory for culture and sensitivity.

\begin{tabular}{ccc}
\hline Specimen referral & Frequency & Percentage (\%) \\
\hline Yes & 19 & 73.1 \\
No & 7 & 26.9 \\
Total & 26 & 100.0 \\
\hline
\end{tabular}

Table 5. Reference laboratories for TB culture and sensitivity specimen.

\begin{tabular}{ccc}
\hline TB reference laboratories & Frequency & Percentage (\%) \\
\hline Research institution (KEMRI Centre for Respiratory Diseases) & 18 & 69.2 \\
A private or commercial laboratory & 1 & 3.8 \\
Any other & 7 & 26.9 \\
Total & 26 & 100.0 \\
\hline
\end{tabular}

The findings in Table 5 illustrate that majority of respondents had been sending their specimens for $\mathrm{TB}$ culture and sensitivity to research institution (KEMRI Centre for Respiratory Diseases) (69.2\%), followed by those who indicated "Any other" thus not sending any specimen (26.9\%) and to private or commercial laboratories (3.8\%). This implies that KEMRI had been receiving specimens from majority of laboratories thus enabling diagnostic capacity for TB.

\section{Discussion}

The results from the study indicated no significant differences in the various laboratory categories in relation to $\mathrm{ZN}$ staining since all laboratories were doing it as well as PCR Restriction Enzyme Assay and Culture and Sensitivity of TB sputum since none of the laboratories did these two tests. However there was statistical significant difference in use of Florescence microcopy $\left[\chi^{2}(1 \mathrm{df}, \mathrm{N}=26)=\right.$ $18.49, \mathrm{p}=0.000<0.05]$ and Gene Xpert Assay $\left[\chi^{2}(1 \mathrm{df}, \mathrm{N}=26)=18.49, \mathrm{p}=\right.$ $0.000<0.05$ ] between public hospitals and private commercial laboratories.

However, a significant differences in the diagnostic capacity of TB between public and private/commercial laboratories was observed in relation to availability of the following resources: Safety cabinet $\left[\chi^{2}(1 \mathrm{df}, \mathrm{N}=26)=18.49, \mathrm{p}=0.000\right.$ $<0.05]$, Centrifuge $\left[\chi^{2}(1 \mathrm{df}, \mathrm{N}=26)=10.64, \mathrm{p}=0.000<0.05\right]$, Gene Xpert resources $\left[\chi^{2}(1 \mathrm{df}, \mathrm{N}=26)=18.49, \mathrm{p}=0.000<0.05\right]$, Freezer/refrigerator $\left[\chi^{2}(1\right.$ df, $\mathrm{N}=26)=14.28, \mathrm{p}=0.000<0.05]$, Incubator $\left[\chi^{2}(1 \mathrm{df}, \mathrm{N}=26)=18.49, \mathrm{p}=\right.$ $0.000<0.05]$ and Auramine $\mathrm{O}$ reagents for TB florescence microscopy $\left[\chi^{2}(1 \mathrm{df}\right.$, $\mathrm{N}=26)=18.49, \mathrm{p}=0.000<0.05]$. The public or government operated facilities or laboratories had enhanced TB diagnostic capacity as compared to the private/commercial laboratories. Research institution (KEMRI Centre for Respiratory Diseases) had been receiving specimens from majority of laboratories thus enabling diagnostic capacity for TB on referral specimens and this was due to lack of referral facilities within Meru County.

The public or government operated facilities or laboratories had enhanced TB 
diagnostic capacity as compared to the private/commercial laboratories. This was evident from significant differences, p-value in relation to referring TB specimens to a TB reference laboratory for culture and sensitivity between the public and private laboratories. Public laboratories made more referrals to a TB reference laboratory making them to have enhanced TB diagnostic capacity as compared to private/commercial laboratories which implies that more efforts needs to be put in private/commercial laboratories to send more referrals to $\mathrm{TB}$ reference laboratories.

The findings of this study are in line with findings from WHO (2010) survey of the availability of TB diagnostic tests at 663 HIV care and treatment facilities from nine Sub Saharan Africa countries, which found that sputum smear microscopy was widely available across the spectrum of healthcare facilities, irrespective of location and type of facility. However, availability of Chest X-ray (CXR), Drug susceptibility testing (DST) and Tubercle bacilli culture were generally limited to secondary and tertiary facilities. The primary health facilities within Meru County needs to be equipped with essential equipment for Chest $\mathrm{X}$-ray services, laboratory requirements for Drug susceptibility and Tubercle bacilli culture testing and availability of trained personnel.

\section{Limitations}

The study did not establish whether TB diagnostic tests to be offered by the medical laboratories under survey, would be accurate or not and did not also establish the frequency of the performance of TB tests or assays over a given period of time and its correlation with the available resources and the population that was served.

\section{Conclusion}

There is need for expansion of TB-related laboratory to lower levels of the health care system so as to meet the expected increase in demand for such services. The efforts should include increasing availability of Chest x-ray (CXR) and Drug Susceptibility Testing (DST) to implement comprehensive TB diagnostic algorithms and ensure timely initiation of treatment for active TB and uptake of IPT for latent TB infection. Strengthening linkages between lower and higher level health facilities for availability of AFB culture and DST, and the improvement in specimen transportation systems will lower threat of MDR/XDR-TB internationally.

\section{Acknowledgements}

We are grateful to Family Health Options Kenya for resource setup for conducting this survey.

\section{Conflicts of Interest}

The authors declare no conflicts of interest regarding the publication of this paper. 


\section{References}

[1] Turner, A.N. (2010) Tuberculosis and Stigmatization: Pathways and Interventions. Public Health Reports, Washington DC, Vol. 125, 34-42. https://doi.org/10.1177/00333549101250S407

[2] Cherry, J., Harrison, G., Kaplan, S., Steinbach, W., Hotez, P., Cruz, A. and Starke, J. (2014) Tuberculosis. In: Cherry, J., Harrison, G., Kaplan, S., Steinbach, W. and Hotez, P. (Ed.), Feigin and Cherry's Textbook of Pediatric Infectious Diseases, Elsevier Saunders, Philadelphia, PA, 1335-1380.

[3] National Institute of Allergy and Infectious Diseases (2012) Tuberculosis. Department of Health and Human Services, USA.

http://www.niaid.nih.gov/topics/tuberculosis/understanding/pages/symptoms.aspx

[4] World Health Organization (2006) Strategic Approach for the Strengthening of Laboratory Services for Tuberculosis Control, 2006-2009. WHO/HTM/TB/2006.364.

[5] Paramasivan, C.N., et al. (2010) Experience Establishing Tuberculosis Laboratory Capacity in a Developing Country Setting. International Journal of Tuberculosis and Lung Disease, 14, 59-64.

[6] World Health Organization, WHO (2014) Global Tuberculosis Report. WHO/HTM/TB.08.

[7] Boehme, C.C., Saacks, S. and O’Brien, R.J. (2013) The Changing Landscape of Diagnostic Services for Tuberculosis. Foundation for New Innovative Diagnostics (FIND), Geneva, Switzerland. Seminars in Respiratory and Critical Care Medicine, 34, 17-31. https://doi.org/10.1055/s-0032-1333468

[8] Ministry of Health, Kenya (2014) Towards Attaining the Highest Standard of Health. Nairobi, 6-7.

[9] World Health Organization, WHO (2014) Estimates of TB and MDR-TB Burden. http://www.who.int/tb/data 